\author{
Bülent Güzel \\ Fatih Cüneyd Korkmaz.
}

http://dx.doi.org/10.21278/brod71101

ISSN 0007-215X

eISSN 1845-5859

\title{
REDUCING WATER ENTRY IMPACT LOADS ON MARINE STRUCTURES BY SURFACE MODIFICATION
}

UDC 629.5.017:629.5.017.21

Original scientific paper

\begin{abstract}
Summary
In this study, the effect of hydrophobicity in reducing the impact forces during the water entry of complex geometries has been demonstrated on bow flare slamming and wet deck slamming. Superhydrophobic surfaces with a contact angle of $160^{\circ}$ and a hysteresis of $3^{0}$ which shows a wetting regime in the Cassie-Baxter state were obtained via a chemical coating. By conducting drop tests with a bow flare ship section model and a catamaran section model at various impact velocities, jet flows, water pileups and air cavities were compared between the hydrophilic and hydrophobic cases via high speed camera images. The impact loads acting on the test bodies under hydrophobic effects were also compared via strain gauge measurements. The rise time of the strain values was measured shorter with smaller magnitudes in the hydrophobic cases. The modification in the jet flow propagation affects the total impact force. The results showed that hydrophobicity modifies the water uprising characteristics and energy balances, and reduces the impact loads acting on marine structures during slamming events.
\end{abstract}

Key words: Hydrophobic; water entry; slamming; impact force; bow flare; wet deck

\section{Introduction}

After a century of research in slamming, predicting impact forces and slamming loads or determining the maximum sustainable speed for ship sailings in different sea states is still the primary aim of many academic and industrial studies. Voluntary speed reductions and course changes are some practical measures taken for preventing slamming related accidents, which cause late arrival times, increased fuel costs. When considering ship slamming in real sea conditions with regular and irregular waves, slamming occurs not as a single event but in a periodic fashion and the rate of this occurrence depends on ship speed and wave height [1]. Slamming can produce forces with a magnitude of the displacement of a ship itself experiencing slamming in irregular sea conditions [2] and cause deadly accidents. Although the slamming impact is short in duration, it can still severely damage a marine structure during the impact and later in the whipping response of the structure following the impact. Yamamoto et al. [3] investigated the damage caused by a strong bow flare slamming on a 
container ship and reported that the peak impact pressure of $840 \mathrm{kPa}$ caused a $10 \mathrm{~m}$-crack on the bottom flare area.

The slamming problem was first studied by von Karman [4] using the momentum theory with the concept of added mass for a seaplane float. The works of von Karman [4] and Wagner [5] have been extended by many researchers by considering some other parameters involved in such water entry events. Since then, most analytical and numerical models of water entry of solid objects have been developed for two types of basic geometrical shapes; straight wedges with constant deadrise angles of $0^{0}$ to $81^{0}$ (flat plates, wedges, cones), and curved wedges with advancing deadrise angles (spheres, cylinders). This strong dependence on the deadrise angle has motivated most of the experimental and numerical studies to focus on the effect of deadrise angle on the impact force and the pressure distribution during the impact, with an emphasize on some hydrodynamic aspects such as impact velocity and drop height. And yet, the peak pressure has little importance in the solution because of its very short duration and low energy content as opposed to the spatially averaged quasi-static pressure distribution assumption. In numerical simulations, the jet flow is cut off and the location of the flow separation is predefined [6,7]. The idealization of the solution of the problem on deadrise angle is due to the high localization of the impact forces acting on the bodies entering vertically into free surface, i.e. peak pressure occurring at the advancing jet root. For the sake of obtaining a prediction of dynamic loads and ship responses, some general simplifying assumptions are made e.g. two-dimensional bodies, no body flexibility, no aeration in flow, no spraying effects, no jet flow or flow separation. Turbulence is not considered because there is not enough time for turbulence to develop in a hull slamming event, although it may significantly influence the impact loads in bow flare and wet deck slamming events because the separated jet flow is turbulent. These assumptions are not adequate to obtain a right solution to the problem, and moreover, there are some other effects necessarily (hydroelasticity, wettability and nonlinearity in free surface) to be considered. Although most studies deal with the rigid bodies, real ship hulls experience flexural deformations. The total kinetic energy of an object at the moment of the first water contact is a function of the entrance velocity. The peak impact force during a slamming event is considered to be the function of the entrance velocity (total kinetic energy). Experimental and numerical results show that the nonlinear effects, when a body penetrates into the free surface, are prominent and play an important role in the hydrodynamics of slamming impacts $[6,7,8]$. Although the pressure inside the jet flow is around the atmospheric pressure and not inducing a significant slamming load, it is still considered to be an indicator about the general phenomena. In the water entry of wedges and cones, the maximum impact pressure occurs at the point where the jet flow is rooted, and the maximum impact force occurs when the jet root is separated from the chine [9]. Whereas, the impact of cylinders and spheres on calm water is characterized by a realization of the maximum impact force at the beginning of the impact [10]. Even though the shape of marine structures are not basic geometries and sea surface is not stationary and disturbed with random waves, estimates of the pressure distribution and the maximum impact forces acting on simple geometries will improve the understanding of the nature of the water entry of solid objects into water, e.g. fore part of a ship. But still, there is a limited number of experimental studies carried out with three-dimensional complex geometries, e.g. slamming loads on a bow flare section.

The wetting regime or the wettability of a solid surface adds another parameter into the slamming phenomenon. Wenzel [11] described the homogeneous wetting regime where the water fills in the micro/nano scale corrugations on a surface, and defined the relationship between roughness and wettability. Wenzel stated that adding surface roughness to Young's relation will increase the wettability caused by the surface chemistry. In a heterogeneous wetting regime described by Cassie-Baxter [12], water particles sit on a mixture of solid and 
air and cannot penetrate into between the corrugations on the solid surface, thus causing air pockets to be trapped under the water particles and resulting in less contact area between the solid surface and the water. When a droplet placed on a superhydrophobic surface, the contact angle measurements taken at the equilibrium of the interfacial tensions of liquid, solid and gas phases read values larger than $150^{\circ}$. Other than the contact angle, another parameter, the rolloff angle (hysteresis) specifies the wetting regime, e.g. homogeneous [11] or heterogeneous [12]. Low hysteresis ensures a stable wetting regime and non-moving contact line [13]. Impact and spreading dynamics of liquids on solid surfaces and the drop deformation depend strongly on the surface properties. Droplet impacting on a solid surface under hydrophobic effects may result in bouncing at low energy impacts depending on the Weber number [14]. In this case, the droplet undergoes an elastic impact (e.g. like an elastic ball) and has an energy storage mechanism [15]. Similar to droplet impact on hydrophobic surfaces, the interaction between a solid object and the free water surface in water entry event may become more elastic with reduced impact forces. In the water entry of spheres, hydrophobic spheres show different characteristics than hydrophilic spheres [16, 17, 18]. Duez et al. [17] showed that the cavity only exists above a threshold velocity, which depends on the wettability, and hydrophobicity promotes early flow separation from the solid surface. Truscott et al. [18] showed that hydrophobicity has strong influence on cavity forming which also affects the net forces acting on the spheres, i.e. impacts with no cavity upon impact have higher force coefficients than cavity-forming impacts. These studies have demonstrated that more kinetic energy is transferred to the bulk water under hydrophobic effects during the impact. Korkmaz and Guzel [19] carried out experiments with cylinders and spheres, and observed significant differences in water uprise characteristics during the impacts of these objects with hydrophobic surfaces. They found that the pressure distribution on the wetted length, the characteristics of the pileups and water jets for flexible and rigid cylinders and spheres change drastically under hydrophobic effects. They also showed that, in water entry of cylindrical and spherical bodies, the amount of kinetic energy transferred to the water is increased under the hydrophobic effects, and the impact loads acting on these objects are decreased. Hydrophobic and superhydrophobic surfaces have gain more attention in marine industry with the rapid evolving of surface modification technologies, i.e. improved coatings, plasma and laser surface modification applications on metals.

In this study, the water entry phenomenon of complex geometries was investigated by carrying out free fall drop tests of a bulbous bow section model and a catamaran section model. Although cylinders and wedges can simply represent the bottom of different ship structures, in order to take the geometric complexity into account in investigating the effects of hydrophobicity on slamming loads, the water entry of real ship geometries needs to be investigated experimentally as well. The effect of hydrophobicity is investigated via high speed camera images and strain gauge measurements. The characteristics of the water entry of hydrophobic coated models are compared with the ones obtained with hydrophilic surfaces.

\section{Water Entry of Simple Geometries; Wedges and Cylinders}

In a free fall of an object impacting the free water surface, potential energy of the object is first transferred to kinetic energy as it accelerates toward the free surface, then some of this kinetic energy is transferred to the fluid during the impact. At the very beginning of the impact, some portion of this transferred energy causes formation of a thin jet flow which climbs up attached to the solid surface. Wagner [5] stated that the maximum pressure always occurs at the pileups near the root of jet flows, and the pressure in this jet flow can be neglected. With further penetration of the solid object, the location of the maximum pressure moves upward on the solid surface following the pileup and the jet root. In the case of 
wedges, the impact force keep building up as the jet root moves along the solid surface and the maximum impact force occurs at the end of the impact, when the jet root is separated from the chine [20]. Whereas in the case of water entry of cylinders and spheres, the momentum transferred to the rising water may cause flow separation from the solid surface depending on the Froude number. The impact of cylinders and spheres on calm water is characterized by a realization of the maximum impact force at the beginning of the impact due to initially zero deadrise angle, and it is strongly related with the location of the separation of the jet flow. Schematic of the water entry of cylinders is depicted in Fig. 1. It compares the free surface elevation and the cavity around a rigid cylinder at low and high energy impacts; left side simulates high energy impacts i.e. high Froude numbers, and the right side simulates the low energy impacts $(F r<3)$ [19]. At low energy impacts, the jet flow climbs up on the solid surface and closes at the back of the cylinder. Whereas, it separates from the cylinder at high energy impacts $(F r>3)$ due to losing its stability (Fig. 1a). There is no impact pressure acting on the cylinder surface after the jet flow is separated from the cylinder [5]. The location of the flow separation varies with the entrance velocity and affects the impact loads [19]. The impact duration is decreased with increasing entrance velocity. As the jet flow separates from the solid surface, it starts forming a cavity around the cylinder and the pileups move outward from the cylinder (Fig. 1b). Larger cavities and larger pileups are observed during the later stages of the impact when the flow separation occurs earlier (Fig. 1c). The forces acting on a solid object during an impact onto the calm free surface are the inertia force $(M g)$, buoyancy force $\left(F_{b}\right)$, skin friction drag and form drag forces $\left(F_{d}\right)$, capillary force $\left(F_{c}\right)$, and the impact force $(F)$ exerted by the fluid on the object. Therefore, the equation of motion of an object entering free water surface can be written as [21]

$$
\begin{aligned}
& \Sigma F=M \ddot{\xi} \\
& M g-F-F_{b}-F_{c}-F_{d}=M \ddot{\xi}
\end{aligned}
$$

where $\xi$ is the penetration depth relative to the still free water surface, $M$ is the mass of the object. The drag forces $\left(F_{d}\right)$ and the buoyancy force $\left(F_{b}\right)$ become important at the later stages of the impact, i.e. after the full submergence of the object. In marine applications and for the experiments carried out with considerably large scale objects, Reynolds number $\left(R e=\rho V_{0} R / \mu\right.$ $\left.>10^{5}\right)$, Froude number $\left(F r=V_{0}^{2} / g R>10^{0}\right)$ and Weber number $\left(W e=\rho V_{0}^{2} R / \sigma>10^{4}\right)$ are so large that fluid viscosity, gravity and surface tension effects on impact loads are neglected in analytical and numerical approaches. The only force acting on the object is the impact force, $F$ (also referred to as slamming force or hydrodynamic force) during the first stages of the impact, e.g. max. impact force is realized at around $R / 6$ submergence depth of a cylinder [19]. This hydrodynamic force, $F$ is applied on the wetted surface of the object. In von Karman's approach, the impact force is associated with the added mass which moves with the object during penetration. In this approach, the added mass gets larger and losses its velocity while penetrating further.

The main difference between von Karman's and Wagner's approaches is the wetted area. In von Karman's theory, the amount of the added mass $\left(m_{a}\right)$ for a 2-D wedge is

$$
m_{a}=\frac{1}{2} \rho \pi c_{0}^{2}
$$

where $c_{0}$ is the wetted width of the wedge when water uprising is neglected. With the use of his flat plate approximation, the wetted length $(c)$ and the impact force $(F)$ can be written as a function of time as [6]

$$
c(t)=\frac{V_{0} t}{\tan \beta} \quad F(t)=\frac{\rho V_{0}{ }^{3} t \pi}{\tan ^{2} \beta}
$$


where $V_{0}$ is the entrance velocity and $\beta$ is the deadrise angle. When Wagner included the water pileup climbing on the wedge surface, the wetted width of the wedge, and thus, the amount of the added mass is increased. Then the equations become

$$
c(t)=\frac{\pi V_{0} t}{2 \tan \beta} \quad F(t)=\frac{\rho V_{0}{ }^{3} t \pi^{3}}{4 \tan ^{2} \beta}
$$
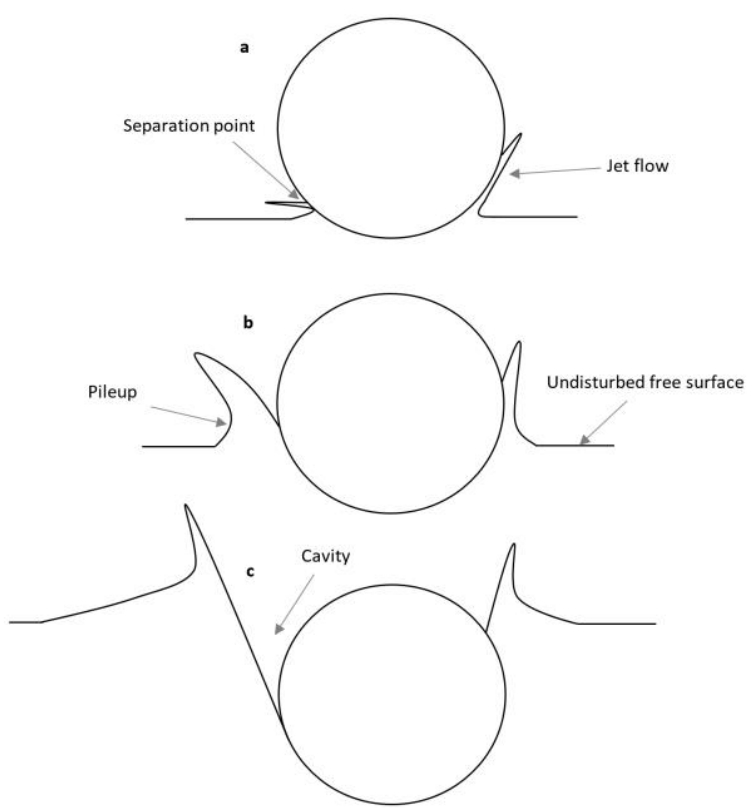

Fig. 1 Schematic of the water entry of a cylinder at high velocities (left) and low velocities (right)

Since then, similar analytical studies have been developed based on the sudden acceleration of this added mass, while the effects of jet flows and pileups are often neglected, and the location of the flow separation and the wetted length are predefined. Under the hydrophobic effects, the water uprising characteristics are modified [17, 18]. And the added mass is a function of the wetted width of an object which depends on the water uprising, and the pressure is distributed on the wetted area. Therefore, the evolution of the wetted surface area in time strongly affects the characteristics and the magnitude of the impact loads [22]. Korkmaz and Guzel [19] experimentally showed that the temporal and spatial pressure variation on the wetted length is related to solid surface characteristics which affects the whole water uprising phenomenon and the location of flow separation that affects the pressure variation on the surface. Although the duration of the peak pressure is very short, its propagating characteristics are different under hydrophobic effects and influence the total impact force. They also showed that hydrophobicity promotes even more energy transfer during the water entry of cylinders and spheres resulting in early flow separation, larger cavities and change in the water rise up characteristics, force distribution and the total slamming forces. Fig. 2 compares the water entry of hydrophilic and hydrophobic cylinders in terms of the characteristics of splashes, jet flows, volume of pileups and air cavities under the same hydrodynamic conditions. In the hydrophilic case, the jet flow steadily climbs on the cylinder's surface and eventually closes from the both side at the back of the cylinder. Whereas in the hydrophobic case, the jet flow loses its stability and separates from the solid surface much earlier. Depending on the contact angle, the volume of pile ups and the size of air cavity are larger and the jet flow is faster in the hydrophobic case comparing to the 
hydrophilic one. The flow separation occurs and air cavities are created at any impact velocity when the contact angle is larger than $150^{\circ}$ [17].

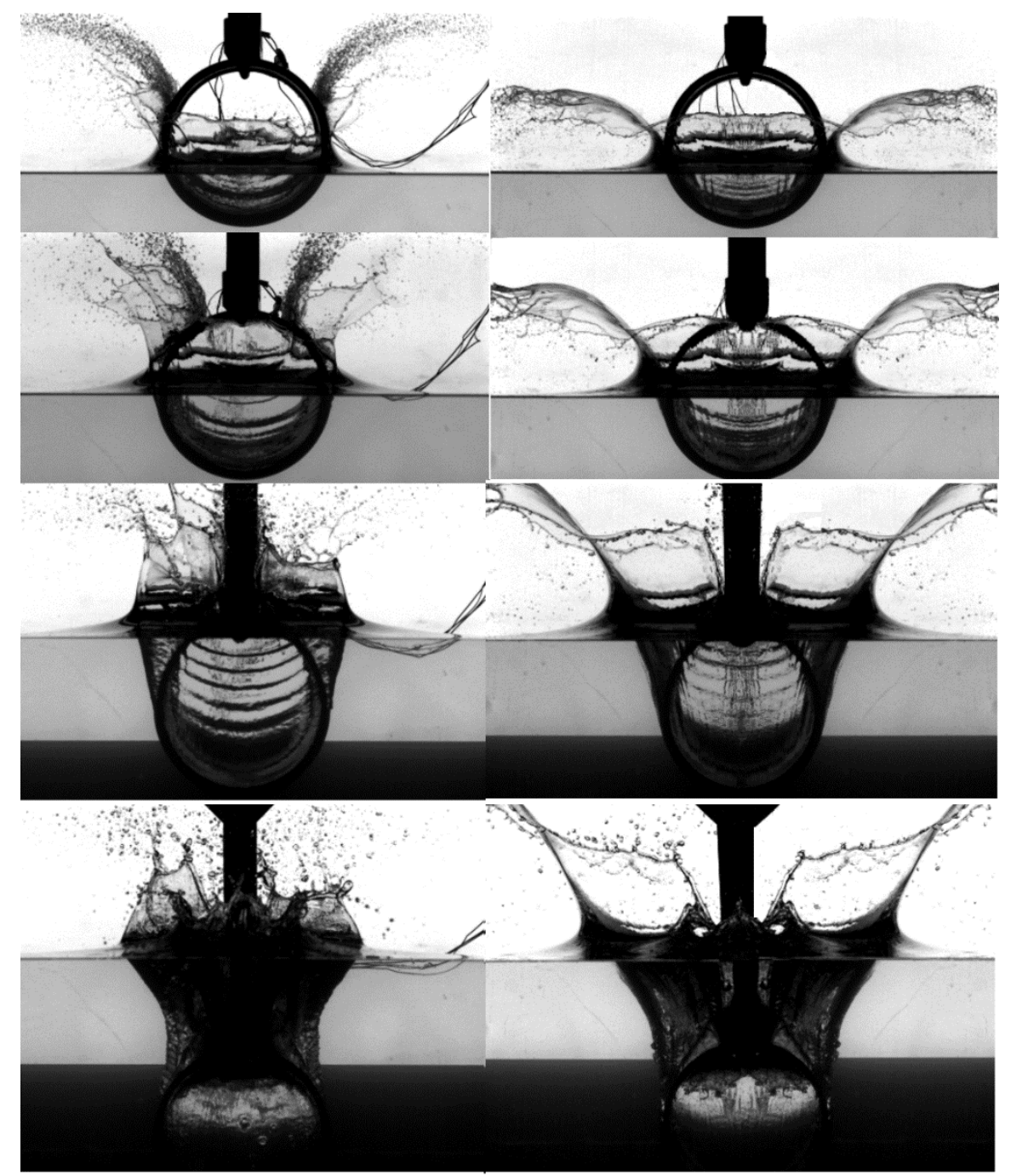

Fig. 2 Photographs taken at different penetration depths $(2 R / 3, R, 2 R, 3 R)$ during the water entry of a cylinder dropped from $15 \mathrm{~cm}$. Left column; hydrophilic surface, Right column; hydrophobic surface $(D=22 \mathrm{~cm}, F r=2.7)$

\section{Experimental Setup}

An experimental setup has been designed and constructed to perform water entry tests by dropping different test objects onto free water surface under gravity. The specimens can be dropped from the heights of $0.05 \mathrm{~m}$ to $4 \mathrm{~m}$ in accordance with the desired impact velocity. The water basin of $1.7 \times 1.0 \times 1.2 \mathrm{~m}$ is built with five acrylic sheets for transparency and reinforced with aluminium profiles. A view of the test setup can be seen in Fig. 3. A vacuum mechanism is added to the setup for holding and releasing the test specimens. The test specimens were manufactured by a 3D printer and made of Polylactic Acid (PLA) with a wall thickness of 2 $\mathrm{mm}$. The geometrical characteristics of the test objects are given in Table 1. In order to ensure symmetric and stable penetration during impacts, a plastering material was used to fill the bottom of the both test models up to $2 \mathrm{~cm}$ high. Hydrophobic surfaces were created by a chemical coating by Wetproof Inc. applied onto the test bodies. This coating is in the order of micrometer in thickness and does not change the dimensions and the mass of the specimens. In order to eliminate the roughness of 3D printing and create similar surface morphology and 
chemical composition, the test models were painted first with an acrylic paint, then coated with the hydrophobic coating material. Both acrylic painting and hydrophobic coating are applied in the same manner with the same number of layers on each test model for creating a similar topology. In order to assess the repeatability of the experiments and the quality and durability of the hydrophobic coating, identical drop tests for the both test models were carried out in different days for three separate coating treatments. Quantitative measurements of the contact angle in the equilibrium state were taken by using Theta optical tensiometer from Attention which uses the sessile drop method.

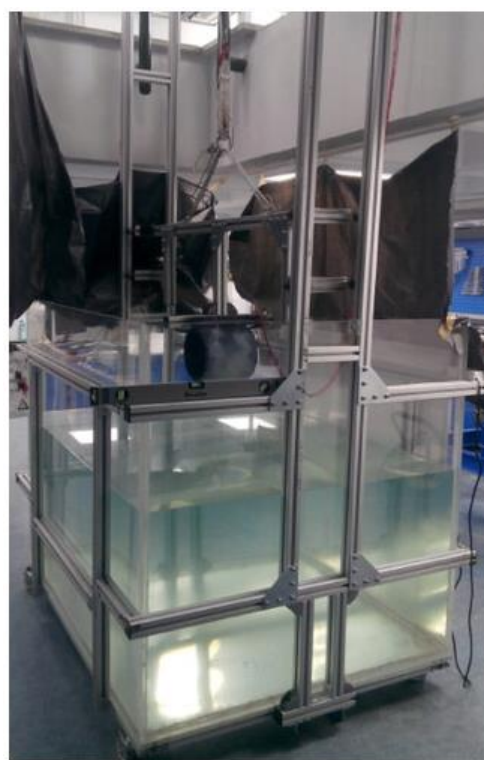

Fig. 3 A view of the experimental setup

In this method, a water droplet is formed in a desired volume and size, and placed on a solid surface, then images of the deposited droplet are recorded using a monochrome video camera at a resolution of $640 \times 488$ pixels at a measuring speed of $60 \mathrm{fps}$ under a cold LED light source preventing water evaporation. Processing and analysing the images with a drop profile detection algorithm (One Attension), static contact angle is determined by fitting Young-Laplace equation around the droplet. $9 \mu \mathrm{l}$ in volume is chosen as the droplet size for the measurements. The measured contact angles vary between $88-90^{\circ}$ on the acrylic painted surface and $148-160^{\circ}$ on the hydrophobic coated surface within an accuracy of $\pm 0.1^{0}$ depending on the painting and coating efficiency, and where it is measured on the coated surface. There is an apparent angle of more than $150^{\circ}$ which shows clear evidence of superhydrophobic behaviour on the coated surfaces. In the contact angle measurements carried out with the tensiometer, it was observed that the water droplets roll off the superhydrophobic coated surface when it is tilted with an angle as small as $3^{0}$. This small contact angle hysteresis shows that the dynamic contact angle is not much different from the static one, which also confirms wetting in the Cassie-Baxter state (Lotus effect).

Table 1 The geometric and the mass values of the test objects

\begin{tabular}{ccc}
\hline \hline & Mass (gr) & Height x Width x Length $(\mathrm{cm})$ \\
\hline \hline Bulbous bow section & 431 & $18.5 \times 15 \times 18$ \\
Catamaran section & 225 & $10 \times 16 \times 16$ \\
\hline \hline
\end{tabular}


In order to understand the physics and dynamics of the impact phenomenon, a Phantom Miro eX4 high speed camera is employed to capture the images of the water entry of the objects at a frame rate of $1400 \mathrm{fps}$ with a spatial resolution equal to $640 \times 480$ pixels. The photos are shot with the help of an LED light system installed on the opposite direction of the camera for a better illumination of the splashes and the air cavity. The deformation of the test bodies during the experiments is measured by water proof strain gauges (Type WFLA-6-113L by Tokyo Sokki Kenkyujo Co. Ltd.) installed on the inner surface of the bulbous bow section and the bottom part of the bow flare section, and on the deck area of the catamaran section. The locations of the strain gauges are shown in Fig. 4. The impact loads acting on the catamaran and bulbous bow sections under hydrophobic effects can be compared via the strain values. A sampling rate of $10 \mathrm{kHz}$ was chosen as an optimum rate for the strain recordings during the impacts. Each test was carried out when the system was free of any wave and vibration to assure repeatability and recorded only when the verticality of impacts was assured.

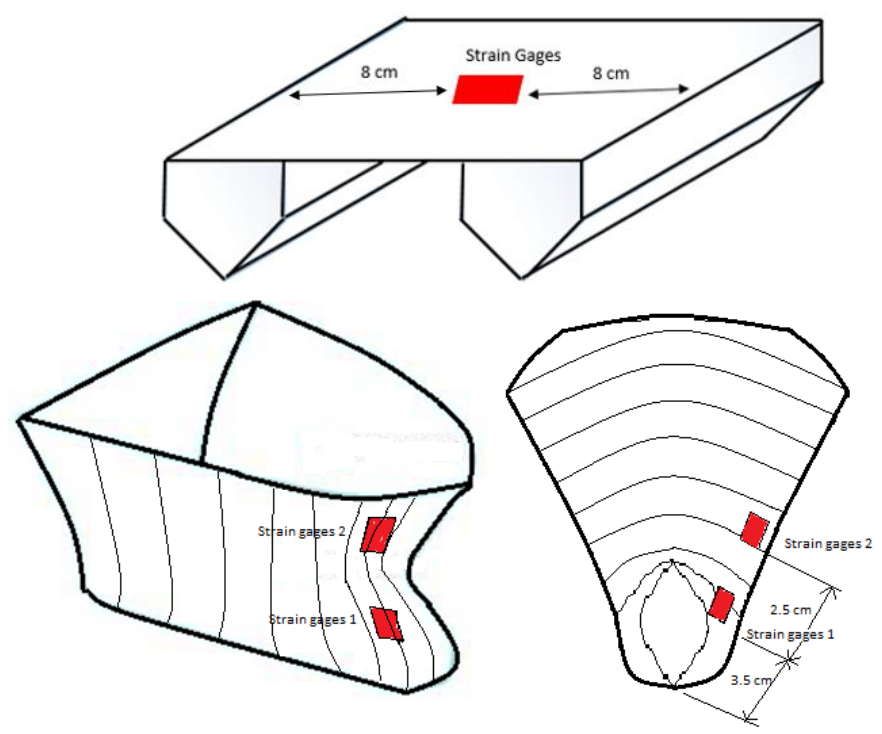

Fig. 4 Location of the strain gauges shown on the technical drawings of the catamaran and bulbous bow sections

\section{Results and Discussion}

During the real slamming events in harsh sea conditions, bow of a ship is lifted out of the water and re-enters into the free water surface. In free fall of the solid objects with small mass ratios onto free water surface, they first accelerate downward due to gravity until piercing the free surface, then slow down as the object penetrates further into water. In reality, bow of a ship is not in a free fall and ship hulls are not rigid. Deformability of the solid objects changes the impact dynamics in favour of experiencing less slamming forces [19, 23]. In order to simulate the fluid flow and the corresponding structural responses of a solid object entering water, and then accurately simulate the real slamming events, the test models need to be elastic in some extent. Moreover, the characteristics of water uprising should be clearly defined and included in the analytical and numerical solutions. Therefore, it is important to carry experiments with realistic ship-like geometries to investigate the water entry phenomenon for complex geometries. On the other hand, the results reported by Korkmaz and Guzel [19] indicate that hydrophobicity may change the characteristics of the water entry phenomenon in bow flare slamming and wet deck slamming in a positive way as well. In order to get an insight on the effects of hydrophobicity on slamming for realistic ship-like 
geometries, free fall drop tests were carried out using a bow flare section model of a container ship and a catamaran section model.

\subsection{Bow Flare Slamming}

While the entrance velocity in free fall tests is determined by the drop height, ensuring the verticality of the entrance, i.e. entering the free water surface with zero roll angle and without any trim, is crucial to maintain the repeatability of the experiments, although the impact force is not increased much until the roll angle of $20^{\circ}[24,25,26]$. The free surface elevation and the cavity formation during a free fall entry of the bow flare section are shown at different time instances in Fig. 5a. $t=0 \mathrm{~ms}$ in this figure represents the time instant when the section model touches the free water surface. Fig. 5b shows the free surface elevation at the same instants under hydrophobic effects. From these images, it can be observed that hydrophobicity certainly changes the characteristics of the impact during the water entry of the bow flare section in a more complicated way. Although the impact occurs initially on the bulb in both cases, water uprising is still larger and faster on the flare section in the hydrophobic case. During the initial stages of the impact, the jet flow separates along the side of the bulb and rises toward the bottom area of the flare and forms a cavity around the flare. The splashes and the jet flows are faster with hydrophobic surfaces and the pileups move outward from the bulb at the beginning of the impact, i.e. cylinder-like impact. Therefore, the jet flow hits at a higher location on the upper part of the bow flare and the cavity becomes larger in the hydrophobic case. In result, the root of the jet flow reaches the chine faster when hydrophobicity is present (Fig. 5b). Fig. 6 shows the time histories of the vertical velocity for the bow flare section dropped from various heights. The vertical velocity of the bow section decreases with time after the impact as it is in free fall with positive buoyancy. It can be seen from these velocity curves that the acceleration at the beginning of the impact is small, then increased and the peak acceleration is observed on the velocity curve at around the time when the jet root and the pileup start hitting the flare area (Fig. 5a2). This effect gets stronger as the entrance velocity is increased.

The bottom of the ship model is normally flatter toward the aft, but the section used in this study is from the bulbous front part which has no flat bottom. The rear and the upper parts of the model section are more like a wedge shape, thus, producing a jet flow and pileup similar to the ones from the water entry of a wedge. On the other hand, the bulb is similar to a cylinder resulting in separation of the jet flow during the impact. This separation promotes air entrapment above the bulb and around the flare. This high pressure air pocket may survive for a longer time during the impact depending on the entrance velocity. While the bulb experiences a bottom slamming and separated jet flow, the free surface elevation causes a bow flare slamming on the flare. The flow separation from the bulb is earlier in the hydrophobic case and causes a weaker bow flare impact because the jet flow hits the flare with a smaller relative angle, thus with a smaller vertical velocity component (Fig. 5a2- $\mathrm{b}_{2}$ ).

Although the strain gauges do not directly measure the impact forces, the strain values are directly proportional to the local stresses, thus correlated with the impact forces. Therefore, they can show the effects of the modified flow separation due to hydrophobicity on the impact loads. Fig. 7 shows the strain time histories from the two strain gauges installed on the bow flare ship section model obtained from the drop height of $50 \mathrm{~cm}$. The impact starts at $t=0 \mathrm{~ms}$. The strain values were recorded only when the roll angle and the trim are fixed at $0^{0}$ during the water entry. The peak strain value occurs when the jet root reaches the flare area (Fig. 5a $\mathrm{a}_{2}-\mathrm{b}_{2}$ ), much before the section model is fully submerged. The peak values of the 
strains are smaller and also build up slower in the hydrophobic case due to larger pileups and faster jet flows. As a result, the impact force reaches its maximum value on a larger wetted area with a smaller magnitude when hydrophobicity is present. It is interesting to observe in the case of the water entry of a three dimensional bow flare ship section that hydrophobicity causes a second, but smaller slam on the bow flare section at the later stages of the impact. This second slam can be seen in Fig. 7 as a second peak value in the history of strains in the hydrophobic case only. This is due to collapsing of the larger cavity formed around the bulb and the flare area in the hydrophobic case; the water slams from all directions onto the bulb and flare area (Fig. 5 $b_{4}-b_{5}$ ). The air pocket is compressed, and with its collapsing, causes a lower second peak pressure and force on the submerged portion of the model. The magnitude of this second slam depends on the characteristics of the cavity. While the cavity is smaller and collapses early in the uncoated case, it is much bigger and survives longer in the hydrophobic case. This second slam is observed at all entrance velocities. The strain gauges start reading the same values after the second slam.

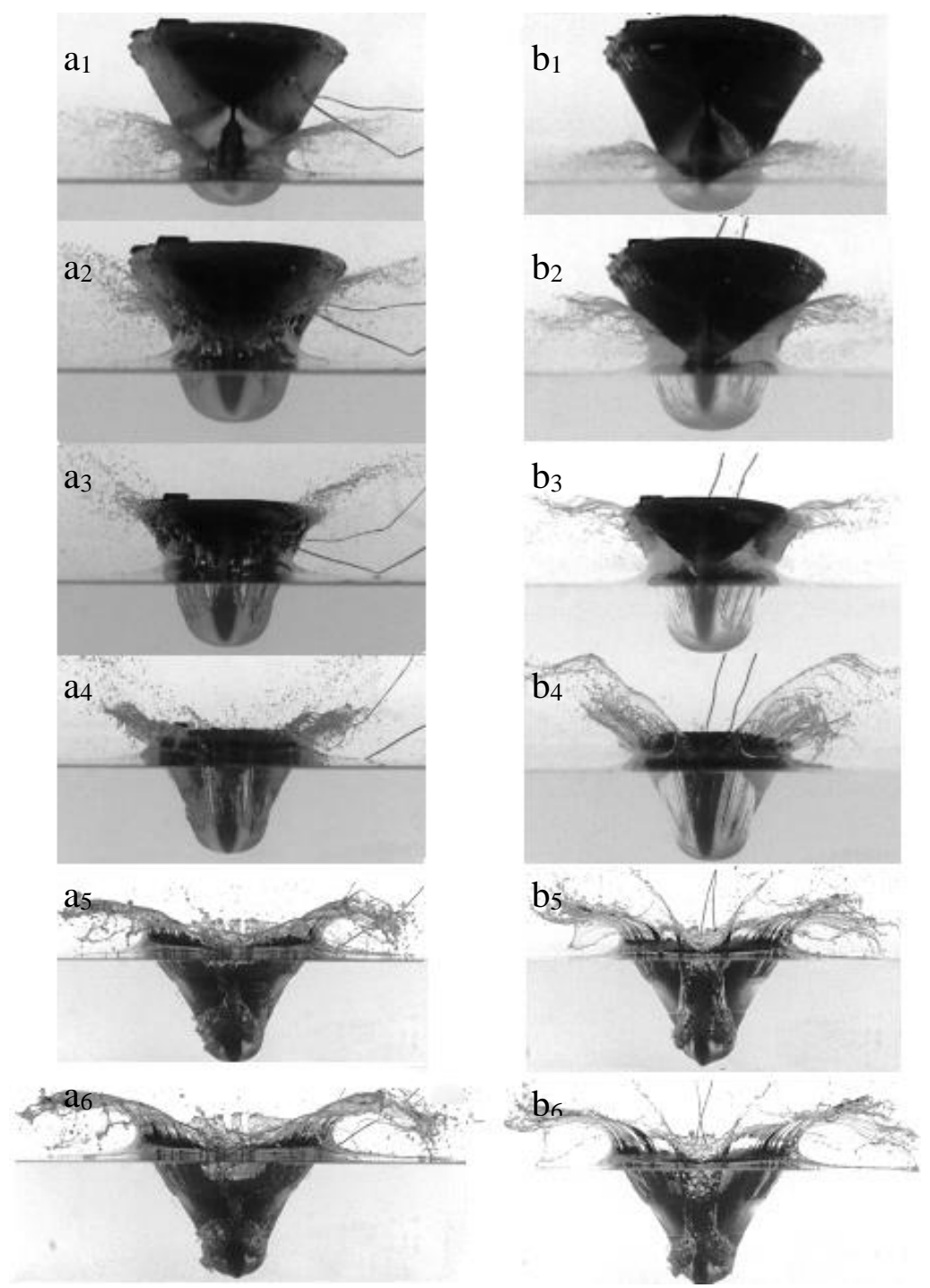

Fig. 5 Photographs taken at different time steps for the water entry of the bow flare ship section model dropped from $50 \mathrm{~cm}$ a) Uncoated case (UC) b) Hydrophobic coated case (HC) $(t=7,14,24,37,66,80 \mathrm{~ms})$ 


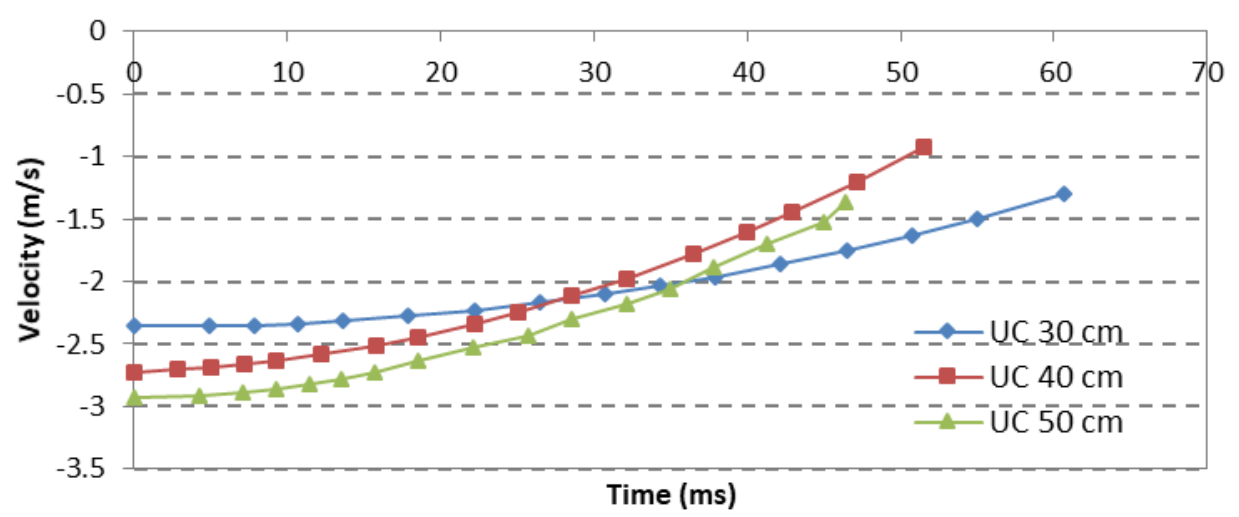

Fig. 6 Time histories of the vertical velocity for the bow flare section dropped from $30-50 \mathrm{~cm}$

Fig. 8 shows the comparison of the peak strain values of the two strain gauges between the uncoated and hydrophobic coated cases at various entrance velocities. The impact loads acting on the bow flare section model as a function of the entrance velocity can be compared through the peak strain values in both cases. The peak strain values of the two strain gauges were always measured smaller with the hydrophobic surfaces comparing to the ones measured with the uncoated surfaces at all entrance velocities. As seen in Fig. 8 that the effect of hydrophobicity is stronger on the strain 2 due to the combined effect of the hydrophobicity on the bulb and the flare area.
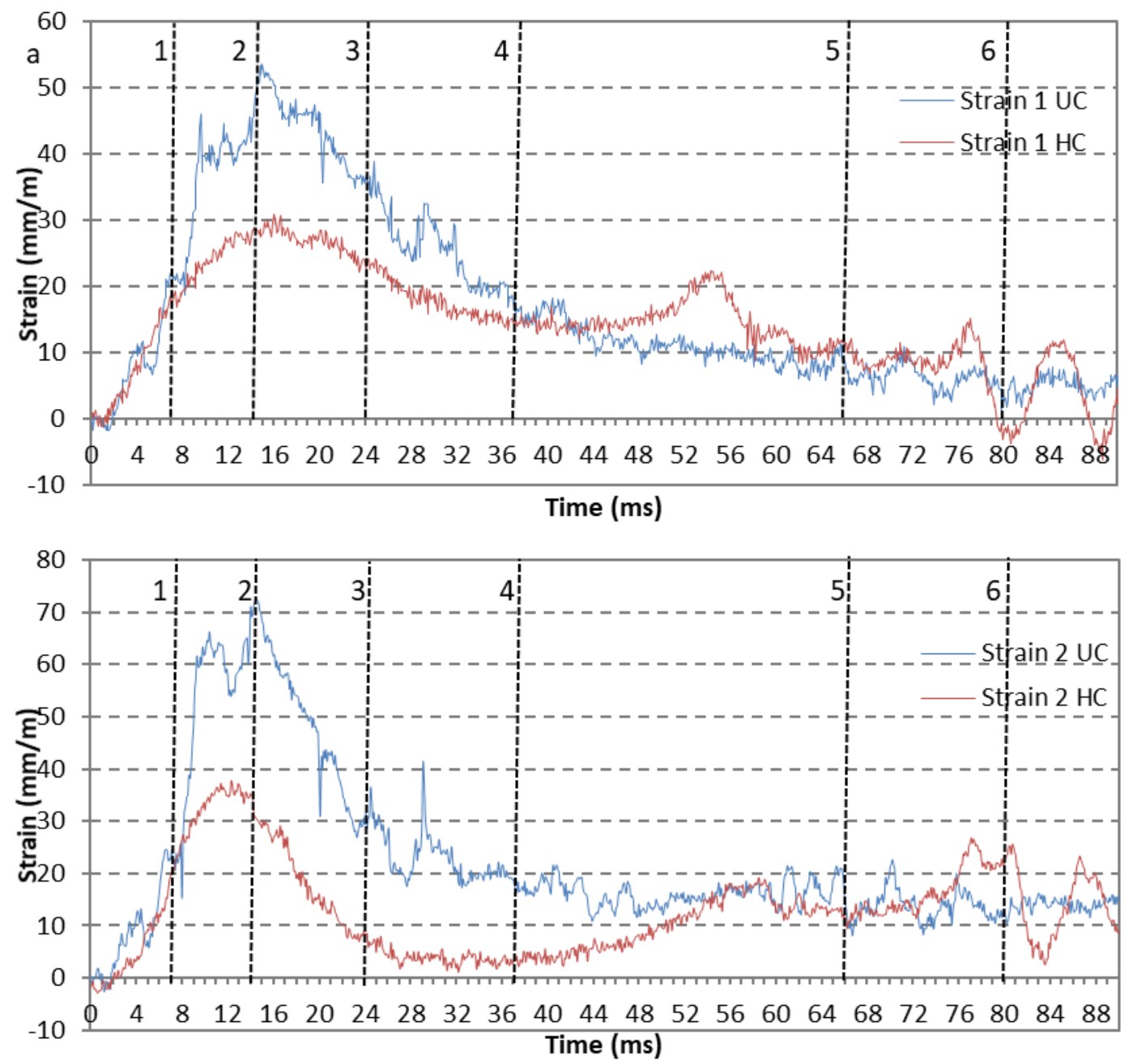

Fig. 7 Time plots of the strain gauges installed on the bow flare section model dropped from $50 \mathrm{~cm}$. 


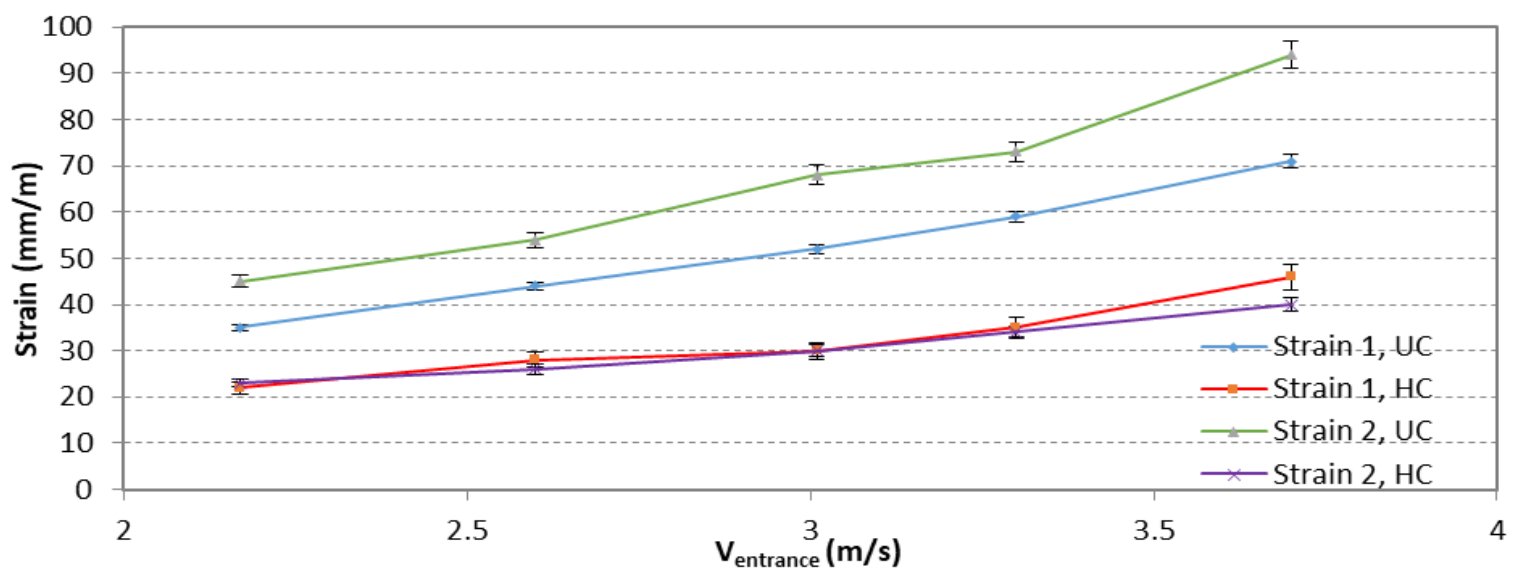

Fig. 8 Peak strain values of the strain gauges installed on the bow flare section model as a function of the entrance velocity. UC; uncoated surface, HC; hydrophobic coated surface

Each peak strain value illustrated in Figs. 8 and 12 is averaged from five identical drop tests. The uncertainty in the mean values of peak strains is statistically calculated as $\pm 2-4 \%$ from the repeated tests and has been represented in terms of error bars in Figs. 8 and 12. The difference in peak strain values between the hydrophobic coated and the uncoated surfaces are much larger than the scatter level of the strain readings.

\subsection{Wet Deck Slamming}

Wedges have different force built-up characteristics than cylinders in a slamming event. In the event of water entry of a wedge into calm water surface, a thin jet flow is generated and move upward along the surface of the wedge, and a large amount of water piles up at the root of the jet is pushed outward as the wedge penetrates further. Wagner [5] described that the maximum pressure always occurs at the pileups near the root of water jet, and the pressure in the jet flow is so small that it can be neglected. During the water entry of wedges, the wetted area and the impact force grow gradually as the object penetrates water [20]. The maximum slamming load occurs when the root of the jet flow leaves the wedge from the chine during the water entry event with constant velocity [26]. There is longer attachment of water as opposed to the water entry of cylinders and spheres in which there is early separation of jet flow with hydrophobic surfaces. The catamaran section used in this study has wedge shape hulls with $30^{\circ}$ deadrise angle. Fig. 9 shows the photographs of the water entry of the catamaran section dropped from $30 \mathrm{~cm}$. At the beginning of the impact, a jet flow is formed and climbs up attached to the hull's surface causing the free surface move upward and outward. While the free surface elevation on the outer sides of the hulls moves freely outward, the jet flows and the pileups on the inner side of the hulls collide (Fig. 9a $a_{2}-b_{2}$ ) filling the cavity between the wet deck and the undisturbed free surface. Two jet flows coming from the both sides hit the bottom of the deck at the same time around the same location (Fig. 9a2). However the jet flows have larger horizontal velocity component (Fig. 9 $b_{1}$ ) and collide in the air before hitting the wet deck in the hydrophobic case. The jet flows reach the wet deck at later times under hydrophobic effects. The time from the first touch of the wedge hulls onto the free water surface to the moment the jet flows hits on the wet deck with the hydrophobic surface is measured to be $40-50 \%$ longer than the one with the uncoated surface. This difference is independent of the entrance velocity, and this elapsed time is linearly decreases with increasing entrance velocity. Larger pileups due to hydrophobicity cause a smaller cavity between the wet deck and the hulls (Fig. 9 $b_{3}$ ). The jet flows with a larger vertical velocity component in the uncoated case leave from the both open end after hitting the wet deck (Fig. $\left.9 a_{3}\right)$. It can be compared between Fig. 9a5- $b_{5}$ that the air cavity around the wedge hull is larger 
on both sides of the hulls in the hydrophobic case. Later, the air trapped (and pressurized) between the wet deck and the pileups is pushed down as the wet deck reaches the undisturbed free surface (Fig. 9a6-b6) and fully submerges.

Fig. 10 shows the time histories of the vertical velocity for the catamaran section dropped from various heights. The vertical velocity of the catamaran section decreases with time after the impact as it is in free fall with positive buoyancy. It can be seen from these velocity curves that the peak acceleration is observed at the beginning of the impact until the root of the jet flow reaches the chine and the wedge hulls are completely immersed (Fig. 9a2). The acceleration is decreased after the jet root leaves the chine. The peak acceleration is larger at larger entrance velocities because the impact loads also gets larger as the entrance velocity is increased.

Wet deck slamming occurs when the wet deck impacts the free water surface. Fig. 11 shows the strain time histories from the strain gauge installed on the catamaran section model obtained from the drop height of $30 \mathrm{~cm}$. The impact starts at $t=0 \mathrm{~ms}$. The first peak strain value occurs due to the wedge hull impact, when the root of the jet flow leaves from the chine (Fig. 9a $a_{2}-b_{2}$ ). This first peak is in the negative direction since the wet deck is bended inward due to hull slamming on both sides. The second peak strain value occurs when the wet deck impacts the free water surface (Fig. 9a5- $b_{5}$ ) and the wet deck is bended outward. Then, the catamaran section keeps oscillating even after it is fully submerged and slows down due to strong buoyancy. The second peak strain values are 10-15\% smaller and build up slower in the hydrophobic case due to the higher waterline between the wet deck and the hulls. The waterline is higher because of the larger pileups. Fig. 12 shows the second peak strain values for the uncoated and hydrophobic coated cases as a function of the entrance velocity. The peak strain values were always measured smaller with hydrophobic surfaces comparing to the ones measured with uncoated surfaces at all entrance velocities. 

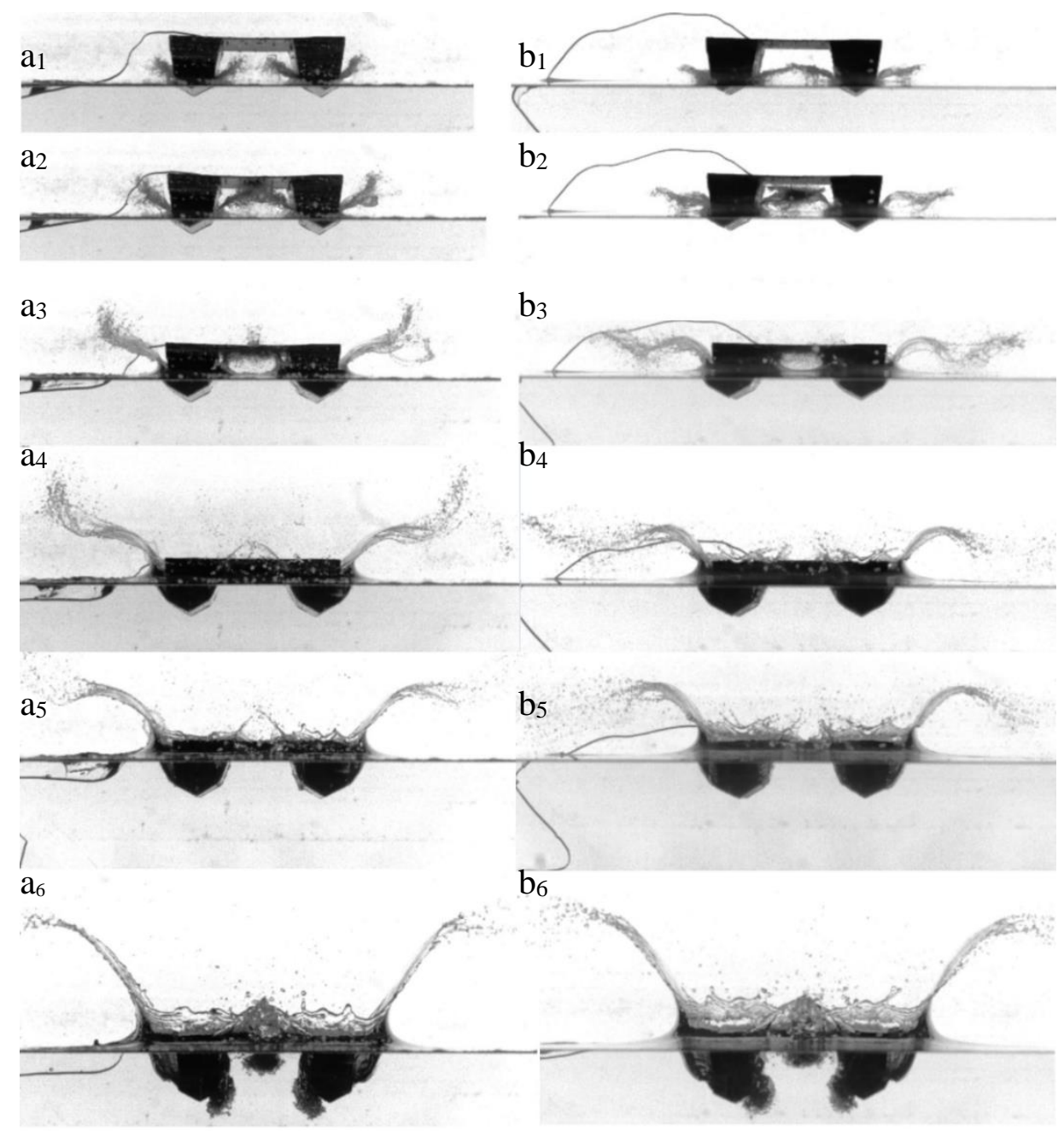

Fig. 9 Photographs taken at different time steps for the water entry of the catamaran section model dropped from $30 \mathrm{~cm} \mathrm{a}$ ) Uncoated case (UC) b) Hydrophobic coated case (HC) $(t=5.6,8.6,14.3,22,30,64.3 \mathrm{~ms})$

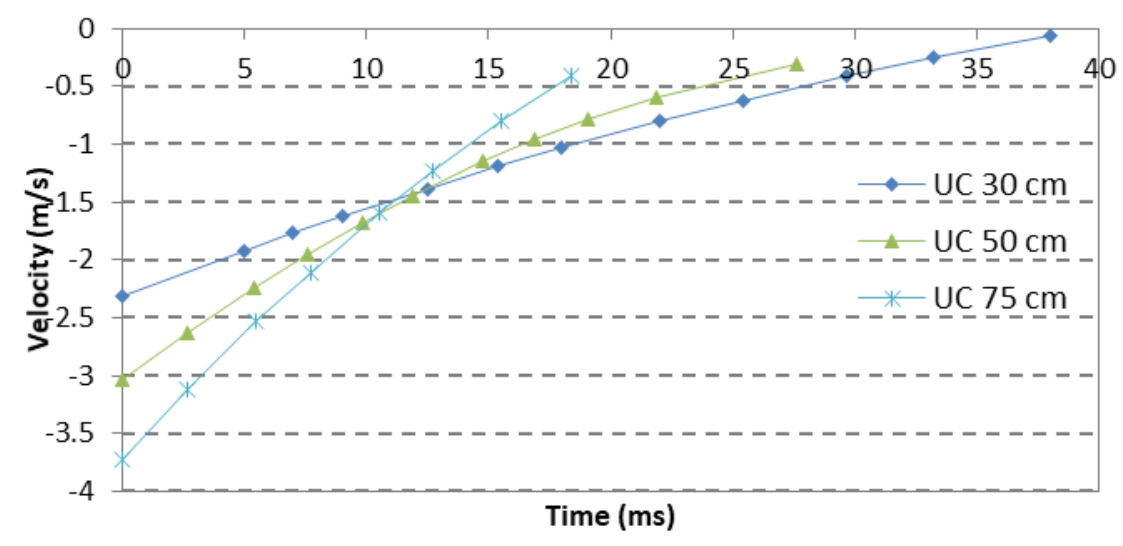

Fig. 10 Time histories of the vertical velocity for the catamaran bow section dropped from $30-75 \mathrm{~cm}$ 


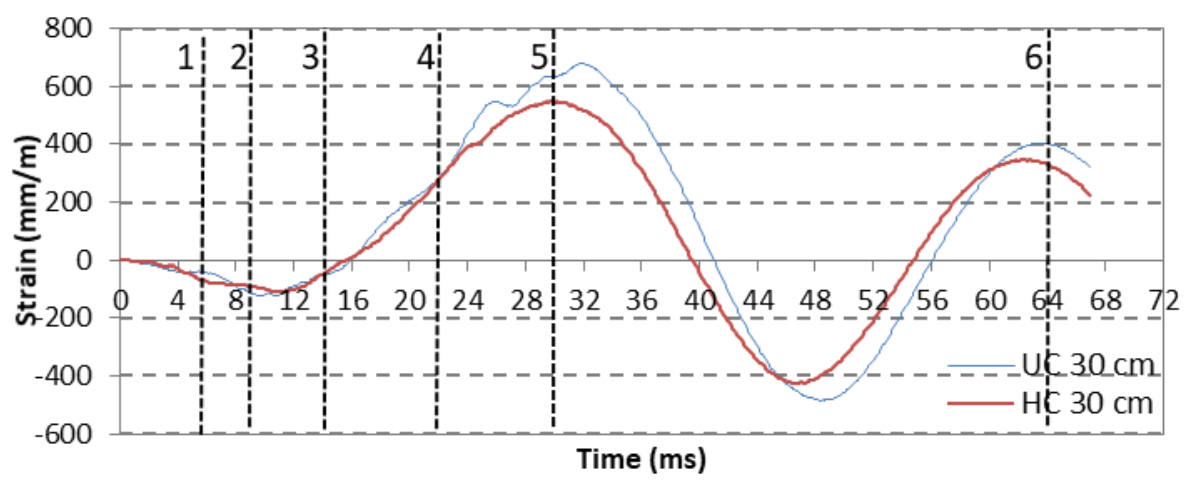

Fig. 11 Time plots of the strain gauges of the catamaran section dropped from $30 \mathrm{~cm}$.

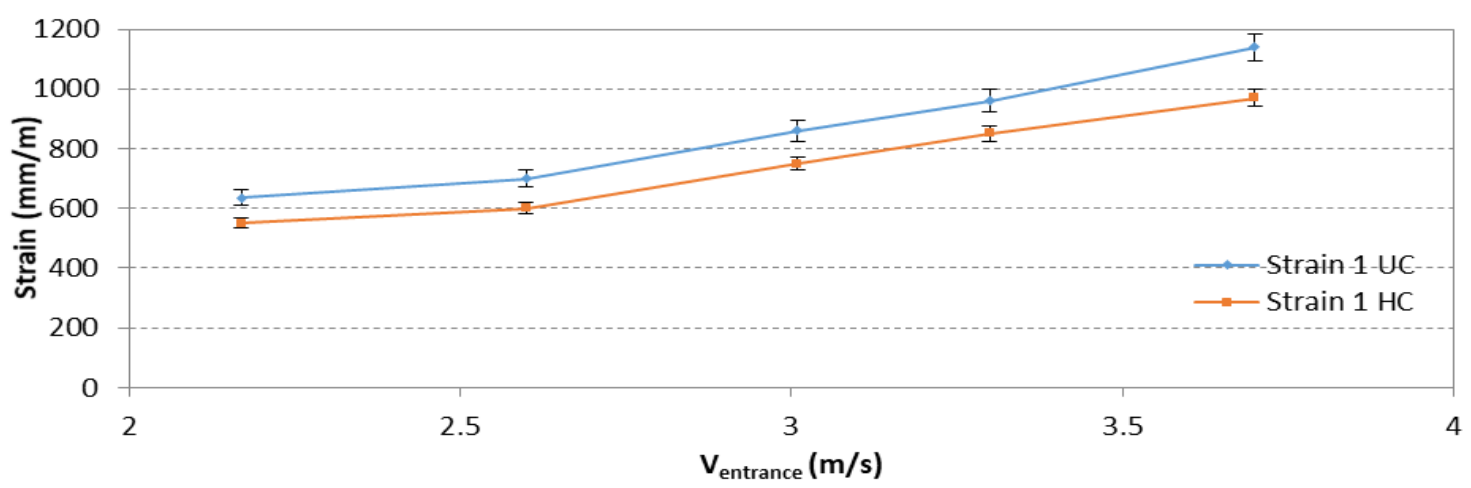

Fig. 12 Peak strain values of the strain gauge installed on the deck of the catamaran section model as a function of the entrance velocity. UC; uncoated surface, HC; hydrophobic coated surface

Hydrophobic surfaces reduce the drag in both laminar and turbulent flows due to slippage caused by the larger contact angles [27]. The surface wetting properties determine the way the liquid connects to the solid to form the contact line in a water entry event $[17,19]$. The first noticeable outcome in any slamming event is that the magnitude of the velocity of the jet generated by the impact is larger than the entrance velocity of the object and increased even to larger values under hydrophobic effects [28]. Therefore, the fast flowing jet flow loses its contact line stability due the effect of slip length on hydrophobic surface. Once the early jet flow separation occurs due to this instability, the jet root and the pileup follow the separated jet flow and cause a larger cavity around the solid object in the hydrophobic case. The wetted length defined by von Karman given in Eqn. 4 neglects the pileup. Whereas, the wetted length defined by Wagner (Eqn. 5) predefines the distance that the pileup reaches as a constant wetting factor of $\pi / 2$. While Wagner's solution overestimates the max. impact force, von Karman's approach underestimates it for rigid bodies [8]. In Wagner's theory, the amount of the added mass for a 2-D wedge is equal to a semi-circle of water with its diameter equal to the wetted width of the wedge with the water pileup climbed on the wedge surface. When hydrophobicity is present, eventhough the wetted length is increased due to faster flowing jet flows and larger pileups, the strain values are decreased (Figs. 8 and 12). In this case, the amount of the added mass is not proportional to the wetted area and decreased with increased wetted width as opposed to the assumptions made in the classical approaches. Other than having the reduced impact loads in bottom slamming [19], early jet flow separation due to hydrophobicity results in reduction on secondary impact loads coming from the separated jet flow interactions as well, i.e. in bow flare slamming and wet deck slamming. Although the jet flow is faster in hydrophobic case, it has a much weaker vertical velocity component which has significant effects in bow flare and wet deck slamming cases. 


\subsection{Scaling Effects}

The effect of hydrophobicity is significant at model scales i.e. early jet flow separation, reduction in impact loads. Ships are subject to gravitational, inertial and frictional forces during ship voyages. In order to have similitude with full scale, a test model has to satisfy the similarity conditions. Therefore, model test results are usually scaled to full scale by using some dimensionless numbers depending on the specific application of ship motions or the ship components. In ship slamming, it is mandatory to have primarily Froude number similarity and secondly Reynolds number similarity between model scale and full scale. But scaling a model according to both Reynolds and Froude numbers similarity is not possible. The Froude number similarity is usually satisfied in hull slamming because this slamming event is dominated by gravitational and inertial forces. Although viscous forces are neglected in hull slamming, Reynolds number similarity may also be significant when considering the jet flow interactions e.g. in bow flare and wet deck slamming.

For the experiments carried out with the test model sections in this study, Reynolds number is in the order magnitude of $10^{5}$ and Froude number is of the order of 1 . Whereas, the Reynolds number for a full scale would be about one to three orders of magnitude larger than the test model section Reynolds number. And for the Froude number, it would be one order of magnitude smaller for a full scale. Therefore, water entry characteristics in model scale under hydrophobic effects can be satisfactorily scaled to full scale since gravitational and viscous effects do not modify significantly the water entry characteristics at model scale with respect to full scale. Moreover, the dimensionless values such as slamming coefficient and pileup coefficient are not affected by scaling effects. On the other hand, the mass ratios, wetted lengths and drop heights need to be scaled-up to full scale values because some scaling effects might arise from turbulence, vorticity and viscous effects since the length and time scales are different at full scale. The scaling effects may influence the separation point of the jet flow, and the flow patterns at model and full scales may be different. The jet flow and the boundary layer before the separation point on solid surface may be turbulent in full scale. Therefore, slamming experiments with small models may be subject to scaling errors. After investigating the hydrophobic effects with small models, water uprise characteristics under hydrophobic effects need to be corrected in scaling to full scale via much larger models with increased Reynolds number and decreased Froude number.

\section{Conclusion}

In this study, the effects of hydrophobic surfaces on the water uprising characteristics and on the impact loads for two different ship models were investigated. Bow flare slamming and wet deck slamming may occur in different sea states or even during fast sailing operations. The water impact on a structure and the relative motion of the jet flow produced from the impact may cause damages on ship structures. It is known that hydrophobicity modifies the free surface elevation in a water entry event of simple geometries like spheres, cylinder and wedges. In this study, impact characteristics of real ship model sections were investigated so as to see the effect of hydrophobicity on complex geometries. The evolution of jet flows and pileups and air cavity formation were observed for two real ship models by using high speed camera images. The peak value of slam induced strains were evaluated and compared between the cases via strain measurements. It was previously observed that the hydrophobicity alters the wetted length which affects the added mass, therefore affects the impact loads in bottom slamming [19]. The hydrophobicity causes the interaction between a ship hull and free water surface to become more elastic. After bottom slamming, the modified 
jet flow alters significantly the jet flow interactions in bow flare and wet deck slamming cases.

In water entry of the bow flare section model, early flow separation from the bulb creates much smaller slamming impact on the flare area and a larger air cavity around it due to larger horizontal velocity component that results in a smaller impact angle between the jet flow and the flare. With hydrophobic surfaces, the peak strain values during water entry are smaller than the ones measured with the uncoated surfaces. Thus, introducing hydrophobicity onto a bow flare section model experiencing water slamming causes a reduction of the peak strains by around $40 \%$ on the flare area.

In water entry of the catamaran section model, larger pileups were observed with hydrophobic surfaces causing a smaller cavity between the wet deck and the hulls. This yields a higher waterline between the wet deck and the hulls resulting in 10-15\% smaller peak strain values on the wet deck in the hydrophobic case.

The strain measurements showed that the rise time of the strain values (i.e. impact forces) is shorter with smaller magnitudes in the hydrophobic cases for both ship section models. The impact loads reduction mechanism in hydrophobic case during bow flare and wet deck slamming events is related to both hydroelasticity of the impact and the modification of the separated jet flow interacting with the upper parts of the ship sections. This study shows that the slamming loads which may cause serious damages on ship structures can be reduced by modifying solid surfaces to be hydrophobic and superhydrophobic.

\section{Acknowledgments}

This research has been financially supported by Yildiz Technical University Research Grants (Grant 2013-1001-KAP03).

\section{REFERENCES}

[1] French BJ, Thomas GA, Davis MR (2015) Slam occurrences and loads of a high-speed wave piercer catamaran in irregular seas. Prc of the Inst of Mech Eng Part M: J of Eng for the Maritime Environ 229(1):45-57. https://doi.org/10.1177/1475090213497705

[2] Thomas GA, Davis MR, Holloway DS, Roberts TJ (2003) Transient dynamic slam response of large high speed catamarans. 7th Int. Conf. on Fast Sea Transportation, Ischia, Italy.

[3] Yamamato Y, Iida K, Fukasawa T, Murakami T, Arai M, Ando A (1985) Structural damage analysis of a fast ship due to bow flare slamming. Int Shipbuild Progr 32:124-136. https://doi.org/10.3233/ISP-1985$\underline{3236902}$

[4] Von Karman (1929) The impact on seaplane floats during landing. NACA TN 321.

[5] Wagner H (1932) Phenomena associated with impact and sliding on liquid surface. Zeitschrift für Angewandte Mathematik und Mechanik.

[6] Zhao R, Faltinsen O (1993) Water-Entry of Two-Dimensional Bodies. J. Fluid Mech 246:593-612. https://doi.org/10.1017/S002211209300028X

[7] Battistin D, Iafrati A (2003) Hydrodynamic loads during water entry of two-dimensional and axisymmetric bodies. J. Fluids Struct 17(5):643-664. https://doi.org/10.1016/S0889-9746(03)00010-0

[8] Cointe R, Armand JL (1987) Hydrodynamic impact analysis of a cylinder. J Offshore Mech Arctic Eng 109(3):237-243. https://doi.org/10.1115/1.3257015

[9] Zhao R, Faltinsen OM, Aarsnes J (1996) Water entry of arbitrary two-dimensional sections with and without flow separation. Procs of 21st Symp. on Naval Hydrodynamics, Norway 408-423.

[10] De Backer G, Vantorre M, Beels C, De Pre J, Victor V, De Rouck J, Blommaert C, Van Paepegem W (2009) Experimental investigation of water impact on axisymmetric bodies. App Ocn Res 31(3):143-156. https://doi.org/10.1016/j.apor.2009.07.003 
[11] Wenzel NR (1936) Resistance of Solid Surfaces to Wetting by Water. Ind Eng Chem 28(8):988-994. https://doi.org/10.1021/ie50320a024

[12] Cassie ABD, Baxter S (1944) Wettability of porous surfaces. Transactions of the Faraday Society 40:546. https://doi.org/10.1039/tf9444000546

[13] He B, Patankar A, Lee J (2003) Multiple equilibrium droplet shapes and design criterions for rough hydrophobic surfaces. Langmuir 19:4999-5003. https://doi.org/10.1021/la0268348

[14] Richard D, Quéré D (2000) Bouncing water drops. Europhysics Letters 50(6):769-775. https://doi.org/10.1209/epl/i2000-00547-6

[15] Clanet C, Beguin C, Richard D, Quere D (2004) Maximal deformation of an impacting drop. J Fluid Mech 517:199-208. https://doi.org/10.1017/S0022112004000904

[16] Duclaux V, Caille F, Duez C, Ybert C, Bocquet L, Clanet C (2007) Dynamics of Transient cavities. J Fluid Mech 591:1-19. https://doi.org/10.1017/S0022112007007343

[17] Duez C, Ybert C, Clanet C, Bocquet L (2007) Making a splash with water repellency. Nat Phys 3:180183. https://doi.org/10.1038/nphys545

[18] Truscott TT, Epps BP, Techet A (2012) Unsteady forces on spheres during free-surface water entry. J Fluid Mech 704:173-210. https://doi.org/10.1017/jfm.2012.232

[19] Korkmaz FC, Guzel, B (2017) Effect of hydrophobicity in slamming; case for advancing deadrise angle. Ocean Eng 129:240-252. https://doi.org/10.1016/j.oceaneng.2016.11.021

[20] Yettou EM, Desrochers A, Champoux Y (2007) A new analytical model for pressure estimation of symmetrical water impact of a rigid wedge at variable velocities. J Fluids Structures 23(3):501-522. https://doi.org/10.1016/j.jfluidstructs.2006.10.001

[21] Abrate S (2013) Hull slamming. Appl. Mech. Rev. 64(6):060803. https://doi.org/10.1115/1.4023571

[22] Korobkin A, Guéret R, Malenica Š (2006) Hydroelastic coupling of beam finite element model with Wagner theory of water impact. J Fluids Structr 22(4):493-504. https://doi.org/10.1016/j.jfluidstructs.2006.01.001

[23] Hao W, Yuan-Sheng C, Da-Ming P, Wei-Wei H, Lin G (2019) Water Entry Hydroelasticity Analysis of Lattice Sandwich Panel with Imperfection:Simulation and Engineering Model. Brodogradnja 70(2):33-59. https://doi.org/10.21278/brod70203

[24] Sun H, Faltinsen OM (2009) Water entry of a bow flare section with a roll angle. J of Marine Sci and Tech 14:69-79. https://doi.org/10.1007/s00773-008-0026-1

[25] Wang S, Soares CG (2013) Slam-induced loads on bow flared sections with various roll angles. Ocean Eng 67:45-57. https://doi.org/10.1016/j.oceaneng.2013.04.009

[26] Yang XB, Xu GD (2018). Numerical simulation of the oblique water entry of wedges with vortex shedding. Brodogradnja 69(4):69-83. https://doi.org/10.21278/brod69406

[27] Daniello RJ, Waterhouse NE, Rothstein JP (2009) Drag reduction in turbulent flows over superhydrophobic surfaces. Phys Fluids 21:085103. https://doi.org/10.1063/1.3207885

[28] Guzel B, Korkmaz FC (2015) Experimental Investigation of Water Entry Impact on Hydrophobic Surfaces. Procs of the 34th Int Conf on Ocean, Offshore and Arctic Engineering 7 https://doi.org/10.1115/OMAE2015-41729

Submitted: $\quad$ 03.06.2019. Bülent Güzel (*corresponding author), bguzel@yildiz.edu.tr

Accepted: $\quad$ 07.01.2020. $\quad \begin{aligned} & \text { Department of Naval Architect } \\ & \text { Yildiz Technical University }\end{aligned}$

Istanbul, Turkey, 34349

Fatih C. Korkmaz, fkorkmaz @yildiz.edu.tr

Department of Marine Engineering Operations

Yildiz Technical University

Istanbul, Turkey, 34349 